\title{
Plasma Proteolytic Cascade Activation during Neonatal Cardiopulmonary Bypass Surgery
}

\author{
Susan A. Maroney ${ }^{1}$ Julie A. Peterson ${ }^{1}$ Wes Zwifelhofer ${ }^{1}$ Nicholas D. Martinez ${ }^{1}$ Ke Yan $^{2}$ \\ Rachel S. Bercovitz ${ }^{1,2,3}$ Ronald K. Woods ${ }^{4,5}$ Alan E. Mast ${ }^{1,6}$
}

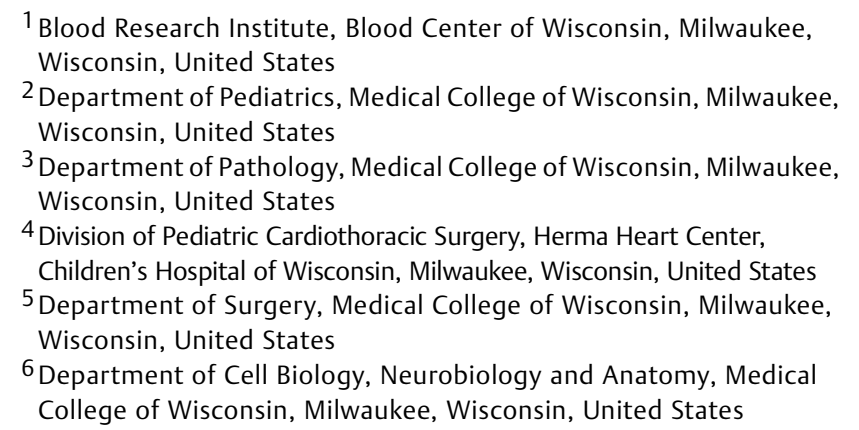

Address for correspondence Alan E. Mast, MD, PhD, Blood Research Institute, Blood Center of Wisconsin, P.O. Box 2178, Milwaukee, WI 53201, United States (e-mail: alan.mast@bcw.edu).

Thromb Haemost 2018;118:1545-1555.

\begin{abstract}
Keywords

- cardiopulmonary

- coagulation

- neonate

- serpin

- TFPI

Background Neonates undergoing cardiopulmonary bypass (CPB) surgery to correct congenital heart defects often experience excessive bleeding. Exposure of blood to artificial materials during CPB may activate coagulation, complement and inflammatory pathways. In addition, the surgical stress placed on the haemostatic system may result in crossactivation of other plasma proteolytic cascades, which could further complicate physiological responses to the surgical procedure and post-operative recovery. Plasma protease inhibitors undergo distinct conformational changes upon interaction with proteases, and, thereby, can serve as endogenous biosensors to identify activation of the different proteolytic cascades. We tested the hypothesis that changes in the concentration and conformation of protease inhibitors regulating plasma proteolytic cascades during neonatal CPB are associated with post-operative bleeding.

Patients and Methods Plasma samples from 44 neonates were obtained at four time points across the surgical procedure. Anti-thrombin, antitrypsin, anti-chymotrypsin, antiplasmin, C1-inhibitor and tissue factor pathway inhibitor (TFPI) concentrations and conformations were evaluated by enzyme-linked immunosorbent assay, transverse urea gradient gel electrophoresis and sodium dodecyl sulphate-polyacrylamide gel electrophoresis.

Results/Conclusion The most striking changes were observed following heparin administration and were associated with the appearance of inactive forms of anti-thrombin and an increase in the plasma concentration of TFPI. Changes in anti-thrombin and TFPI remained evident throughout surgery and into the post-operative period but were not different between patients with or without post-operative bleeding. The concentration of antitrypsin decreased across surgery, but there was no significant accumulation of inactive conformations of any inhibitor besides anti-thrombin, indicating that widespread cross-activation of other plasma proteolytic cascades by coagulation proteases did not occur.
\end{abstract}

received

May 1, 2018

accepted after revision June 8, 2018 (c) 2018 Georg Thieme Verlag KG Stuttgart . New York
DOI https://doi.org/ 10.1055/s-0038-1667198. ISSN 0340-6245. 


\section{Introduction}

Cardiac surgery is a common cause of severe bleeding in neonates. ${ }^{1}$ Cardiopulmonary bypass (CPB) is routinely used to isolate and support the cardiopulmonary system during complex cardiac procedures and places different stressors on neonatal haemostasis than occur in adults undergoing CPB. These include a larger relative prime volume, obligate use of donor blood and more extensive haemodilution of plasma proteins. ${ }^{2}$ The neonatal haemostatic system also undergoes multifactorial change during $\mathrm{CPB}$, including alterations of platelet number and function, ${ }^{3-5}$ dilution of plasma growth factors, such as thrombopoietin, ${ }^{3}$ and alteration in plasma coagulation proteins.

The neonatal haemostatic system is altered during CPB by transfusion of blood products collected from adults and teenagers to prime the bypass pump and treat surgical bleeding. Maternal coagulation proteins do not cross the placenta, ${ }^{6}$ and therefore, the plasma concentrations of neonatal coagulation proteins tend to be lower than in adults. ${ }^{7,8}$ Pro-coagulant factors II, VII, IX, X, XI and XII are particularly low at birth, ranging from 30 to $50 \%$ of adults. ${ }^{7,8}$ Anticoagulant plasma proteins, protein $\mathrm{C}$, protein $\mathrm{S}$, and antithrombin are also below $50 \%$ of adult concentrations at birth, ${ }^{7,8}$ producing some balance between the pro- and anticoagulant potential of neonatal plasma. ${ }^{9}$ Likewise, neonatal concentrations of proteins modulating plasma protease cascades including C4b-binding protein and plasminogen are reduced. $^{7,8}$ Transfusion of blood products to prime the bypass pump and maintain haemostasis shifts the neonatal circulation towards that of an adult, which alters the balance between pro- and anticoagulant proteins in neonatal plasma. This balance is further altered by heparin administration during $\mathrm{CPB}$, and its subsequent reversal with protamine.

Exposure of blood to artificial materials in the bypass pump can cause contact activation of plasma coagulation, complement and inflammatory cascades. ${ }^{10,11}$ Tissue factor-dependent coagulation and inflammation, as a result of monocyte activation and surgical trauma, also occur during CPB. ${ }^{12-15}$ In addition, coagulation proteases can cross-activate other plasma proteolytic cascades and vice versa. ${ }^{16,17}$ Thus, the neonate undergoing CPB is susceptible to widespread and unregulated proteolysis with consumption of plasma protease inhibitors that regulate blood coagulation, fibrinolysis, complement and inflammation. Members of the serpin superfamily of protease inhibitors undergo large and distinct conformational changes when they interact with proteolytic enzymes. ${ }^{18-20}$ These occur through structural transition of the active site loop as it partially or fully inserts into a large $\beta$-sheet, altering thermodynamic stability of the serpin. ${ }^{19}$ Serpin conformations include the virgin form, which is the active inhibitor and is cleared slowly from plasma; the cleaved form, in which the reactive site loop is proteolytically inactivated and also is cleared slowly from plasma; ${ }^{19}$ and the protease-complexed form, which is an equimolar complex of the serpin and a target protease and is more rapidly cleared from plasma. ${ }^{19,21}$ We sought to utilize serpin conformational changes that occur during neonatal $\mathrm{CPB}$ as 'endogenous bio- sensors' to detect activation of the different plasma proteolytic cascades during the surgical procedure. To this end, we examined the concentration and the conformational stability of the serpins anti-thrombin, antitrypsin, anti-chymotrypsin, anti-plasmin and C1-inhibitor, as well as the Kunitz-type protease inhibitor, tissue factor pathway inhibitor (TFPI), in plasma samples obtained at different time points across the course of neonatal CPB surgery and correlated the measurements with bleeding outcomes.

\section{Materials and Methods}

\section{Study Design}

Neonatal patients requiring cardiac surgery with CPB were enrolled after obtaining informed parental consent. Exclusion criteria were birth weight under $2.5 \mathrm{~kg}$, known inherited haemostatic abnormality, extracorporeal membrane oxygenation at the time of surgery or history of previous CPB. The study was approved by the Children's Hospital of Wisconsin Institutional Review Board.

\section{Sample Collection}

Blood was collected from arterial lines that were infused with $1 \mathrm{unit} / \mathrm{mL}$ heparin at 1 to $2 \mathrm{~mL} /$ hour to prevent blood from clotting in the line. Before each blood sample collection, the first 1 to $2 \mathrm{~mL}$ of blood was discarded to remove the heparin in the line before drawing the test sample. Blood was collected into $3.2 \%$ sodium citrate at four time points. The samples were as follows: baseline, admittance to the operating room; on$\mathrm{CPB}$, following heparin administration and the start of $\mathrm{CPB}$, aortic cross-clamp removal and prior to platelet or cryoprecipitate transfusion; post-CPB, after patient removal from $\mathrm{CPB}$, completed rewarming and protamine administration; and post-op, upon cardiac intensive care unit admission. Each sample was $0.5 \mathrm{~mL}$ whole blood which was transported to the research laboratory, spun at $2,500 \times g$ for 10 minutes at $25^{\circ} \mathrm{C}$, and plasma stored at $-80^{\circ} \mathrm{C}$.

\section{Surgical Haemostatic Management and Transfusion}

Bypass pump prime $(450 \mathrm{~mL})$ was administered as fresh whole blood ( $<2$ days storage) or a 1:1 ratio of packed red blood cells (PRBCs) and fresh frozen plasma (FFP) when fresh whole blood was not available. All neonates received tranexamic acid as a $30 \mathrm{mg} / \mathrm{kg}$ loading dose, followed by $10 \mathrm{mg} / \mathrm{kg} /$ hour infusion to inhibit fibrinolysis during surgery. In addition, $0.1 \mathrm{mg} / \mathrm{mL}$ tranexamic acid was added to the CPB prime. Heparin was dosed to maintain the activated clot time above 480 seconds. An institutional transfusion strategy for neonatal congenital cardiac surgery with CPB was applied throughout the study. Immediately prior to termination of $\mathrm{CPB}$, all neonates received one-fourth unit of single donor apheresis platelets and 1 unit of cryoprecipitate. After CPB, protamine, one-fourth unit of the same single donor apheresis platelets and PRBCs were administered. Beyond this uniform protocol, neonates were transfused to maintain haematocrit above $35 \%$, platelet count over $100 \times 10^{3} / \mu \mathrm{L}$ and fibrinogen above $200 \mathrm{mg} / \mathrm{dL}$. Modified ultrafiltration was performed uniformly in all neonates at the end of CPB. 


\section{Post-Operative Bleeding Definition}

A standardized definition of post-operative bleeding was used as follows: total chest tube output $\geq 84 \mathrm{~mL} / \mathrm{kg}$ during the first 24 post-operative hours ( $\geq 3.5 \mathrm{~mL} / \mathrm{kg} /$ hour average); $\geq 7 \mathrm{~mL} / \mathrm{kg} /$ hour chest tube output for $\geq 2$ consecutive hours during the first 12 post-operative hours; and re-exploration for bleeding or cardiac tamponade physiology during the first 24 post-operative hours. ${ }^{22}$

\section{Fibrinogen, D-Dimer and Total Protein Assays}

Fibrinogen was measured using the STA Fibrinogen 5 Kit and Ddimer was measured using the STA Liatest D-Di (Diagnostica Stago, Inc, Parsippany, New Jersey, United States). Plasma total protein was measured using the bicinchoninic acid protein assay (ThermoScientific, Waltham, Massachusetts, United States).

\section{Concentration of Plasma Protease Inhibitors}

Enzyme-linked immunosorbent assay (ELISA) assays measured the plasma concentrations of anti-thrombin, antitrypsin, anti-plasmin, C1-inhibitor and TFPI. Serpin assays were performed as recommended by the manufacturer, C1-inhibitor and anti-plasmin (Sino Biological, Beijing, China); and antitrypsin and anti-thrombin (Enzyme Research Laboratories, South Bend, Indiana, United States). TFPI ELISA assays measuring total TFPI and TFPI $\alpha$ were developed in our laboratory. ${ }^{23,24}$ The results of the assays were recorded as $\%$ adult normal plasma, based on comparison to the normal pooled plasma control tested on each plate.

\section{Transverse Urea Gradient Gel Electrophoresis}

Transverse urea gradient (TUG) gels containing 7\% polyacrylamide and a continuous horizontal linear 0 to $8 \mathrm{M}$ urea gradient within an ammediol buffer system were performed under non-denaturing conditions. ${ }^{19,20}$ Each gel was loaded with $5 \mu \mathrm{L}$ of plasma diluted into $145 \mu \mathrm{L}$ sample buffer. Proteins were visualized by Western blot.

\section{Sodium Dodecyl Sulphate-Polyacrylamide Gel Electrophoresis}

Plasma TFPI ligand pull-down using factor Xa (FXa) beads were performed as previously described. ${ }^{25}$ Briefly, FXa beads was added to $50 \mu \mathrm{L}$ of plasma, rotated at $4^{\circ} \mathrm{C}$ for 1 hour and washed with phosphate-buffered saline (PBS) 0.05\% Tween-20 followed by PBS. Washed beads were boiled in sodium dodecyl sulphate (SDS) sample buffer and the supernatant was loaded onto a 4 to $20 \%$ continuous gradient SDS-polyacrylamide gel electrophoresis (SDS-PAGE) gel.

\section{Western Blotting}

Proteins separated on the TUG or SDS-PAGE gels were transferred to polyvinylidene difluoride (TUG) or nitrocellulose (SDS-PAGE) membrane at 400 mAmps for 1 hour. The membranes were washed in PBS, blocked for 1 hour and incubated overnight at $4^{\circ} \mathrm{C}$ with polyclonal antibodies against antitrypsin (Millipore, Temecula, California, United States), anti-plasmin (Sino Biological), anti-chymotrypsin (Proteintech, Chicago, Illinois, United States), anti-thrombin (Enzyme Research Laboratories) and C1-inhibitor (Cedarlane, Burlington, North
Carolina, United States), then washed and probed with secondary antibodies for 1 hour at room temperature. Blots were imaged using a LI-COR Odyssey Imaging System (Licor, Lincoln, Nebraska, United States).

\section{Statistical Methods}

To account for repeated measures within each subject, a generalized estimating equation with an autoregressive covariance structure was used to compare bleeders and nonbleeders. Either a normal distribution with identity link function or a gamma distribution with log link function was specified depending on the outcome. A Bonferroni stepdown (Holm) correction method was used to adjust for multiple comparisons. A $p$-value of $<0.05$ was considered as statistically significant. SAS 9.4 was used for the analyses.

\section{Results}

\section{Study Population}

This prospective observational study enrolled 44 term neonates $<30$ days of age (median: 7.0 days) undergoing surgical repair of congenital heart defects with CPB. There were 20 females and 24 males with median weight of $3.3 \mathrm{~kg}$. The cohort had a mean Risk Adjusted Classification for Congenital Heart Surgery-1 (RACHS-1) score of 4, reflecting high complexity of the cardiac procedures.

\section{Post-Operative Bleeding Assessment}

Of the 44 neonates, 16 (36\%) met the criteria for post-operative bleeding. Population comparisons were performed between the non-bleeder and bleeder groups ( - Table $\mathbf{1}$ ). There were no differences found in age at surgery, birth weight or height or total time on CPB. There were no difference in RACHS-1 score, and the number of each procedure type by group is presented in -Table 1. There were no differences in baseline platelet count, haematocrit, pro-thrombin time, activated partial thromboplastin time, time on $\mathrm{CBP}$, heparin level while on CPB or the initial protamine administered to reverse heparin between groups. The bleeder group experienced more chest tube output in the first 24 hours following surgery (294 vs. $115 \mathrm{~mL}$ ). The bleeder group was given approximately twofold (330 vs. $152 \mathrm{~mL}$ ) more average total combined volume of blood products (FFP, PRBC, platelets, cryoprecipitate) during surgery than was the non-bleeder group. This difference was largely accounted for by greater amount of $\operatorname{PRBC}(p=0.0009)$ and FFP $(p=0.002)$ transfused in the bleeder group, while amounts of platelet and cryoprecipitate were equal between groups (-Table 1).

\section{Change in Surgical Total Plasma Protein}

Blood products collected from adults and/or teenagers were used to prime the bypass pump in amounts exceeding the total blood volume of the neonate. This increased the total plasma protein concentration across the surgical procedure (-Fig. 1). Baseline plasma protein averaged $60 \%$ than that of adults, increased to $89 \%$ on-CPB, further increased to $98 \%$ post-CPB and then decreased to $83 \%$ post-op. Despite differences in amounts of blood products transfused, there was no 
Table 1 Demographics, surgical procedures, clinical data and transfusion of blood products in the non-bleeder and bleeder groups

\begin{tabular}{|c|c|c|c|}
\hline & Non-bleeder & Bleeder & \\
\hline Gender & & & $p=0.28$ \\
\hline Male & 17 & 7 & \\
\hline Female & 11 & 9 & \\
\hline Age at surgery (d) & $7.82 \pm 4.24$ & $7.0 \pm 5.44$ & $p=0.47$ \\
\hline Birth weight (kg) & $3.42 \pm 0.44$ & $3.25 \pm 0.49$ & $p=0.25$ \\
\hline Birth height $(\mathrm{cm})$ & $50.66 \pm 3.12$ & $49.34 \pm 2.16$ & $p=0.14$ \\
\hline Race & & & $p=0.086$ \\
\hline White & 23 & 9 & \\
\hline Non-white & 5 & 7 & \\
\hline \multicolumn{4}{|l|}{ Surgical procedure type } \\
\hline RACHS-1 & $3.82 \pm 2.04$ & $4.93 \pm 1.33$ & $p=0.25$ \\
\hline Norwood & 11 & 9 & \\
\hline $\mathrm{ASO} \pm \mathrm{VSD}$ & 8 & 0 & \\
\hline $\mathrm{IAA} \pm \mathrm{VSD}$ & 1 & 2 & \\
\hline TOF & 1 & 2 & \\
\hline Central shunt & 1 & 1 & \\
\hline TAPVR & 1 & 1 & \\
\hline VSD & 2 & 0 & \\
\hline AP window & 1 & 0 & \\
\hline DORV & 1 & 0 & \\
\hline Truncus arteriosus & 0 & 1 & \\
\hline ASD & 1 & 0 & \\
\hline \multicolumn{4}{|l|}{ Clinical data } \\
\hline Baseline platelet count $\left(10^{3} / \mu \mathrm{L}\right)$ & $291.36 \pm 125.74$ & $244.73 \pm 118.13$ & $p=0.24$ \\
\hline Baseline haematocrit & $42.82 \pm 6.03$ & $42.45 \pm 6.14$ & $p=0.11$ \\
\hline Baseline PT & $16.30 \pm 1.44$ & $15.59 \pm 0.83$ & $p=0.087$ \\
\hline Baseline aPTT & $63.56 \pm 40.44$ & $47.04 \pm 8.54$ & $p=0.062$ \\
\hline CPB time (min) & $196.18 \pm 74.71$ & $244.56 \pm 163.85$ & $p=0.55$ \\
\hline Heparin level on CPB (IU/mL) & $3.32 \pm 1.06$ & $3.5 \pm 1.4$ & $p=0.50$ \\
\hline Initial protamine administered & $24.89 \pm 12.51$ & $23.67 \pm 9.5$ & $p=0.93$ \\
\hline Average chest tube output within first $24 \mathrm{~h}(\mathrm{~mL})$ & 115 & 294 & \\
\hline \multicolumn{4}{|l|}{ Blood products } \\
\hline WB prime & 11 & 6 & \\
\hline PRBC:FFP prime & 14 & 10 & \\
\hline WB:FPRBC:FP prime & 3 & & \\
\hline Non-prime FFP (mL) & $45 \pm 100$ & $138 \pm 219$ & $p=0.002$ \\
\hline Non-prime PRBC (mL) & $56 \pm 134$ & $133 \pm 216$ & $p=0.0009$ \\
\hline Non-prime platelets (mL) & $41 \pm 52$ & $47 \pm 44$ & $p=0.3235$ \\
\hline Non-prime cryoprecipitate (mL) & $10 \pm 16$ & $12 \pm 17$ & $p=0.2975$ \\
\hline
\end{tabular}

Abbreviations: AP, aortopulmonary; aPTT, activated partial thromboplastin time; ASD, atrial septal defect; ASO, arterial switch operation; CPB, cardiopulmonary bypass; DORV, double outlet right ventricle; FFP, fresh frozen plasma; FP, frozen plasma; FPRBC, frozen packed red blood cell; IAA, interrupted aortic arch; PRBC, packed red blood cell; PT, pro-thrombin time; RACHS-1, Risk Adjusted Classification for Congenital Heart Surgery-1; TAPVR, total anomalous pulmonary venous return; TOF, Tetralogy of Fallot; VSD, ventricular septal defect; WB, whole blood. 


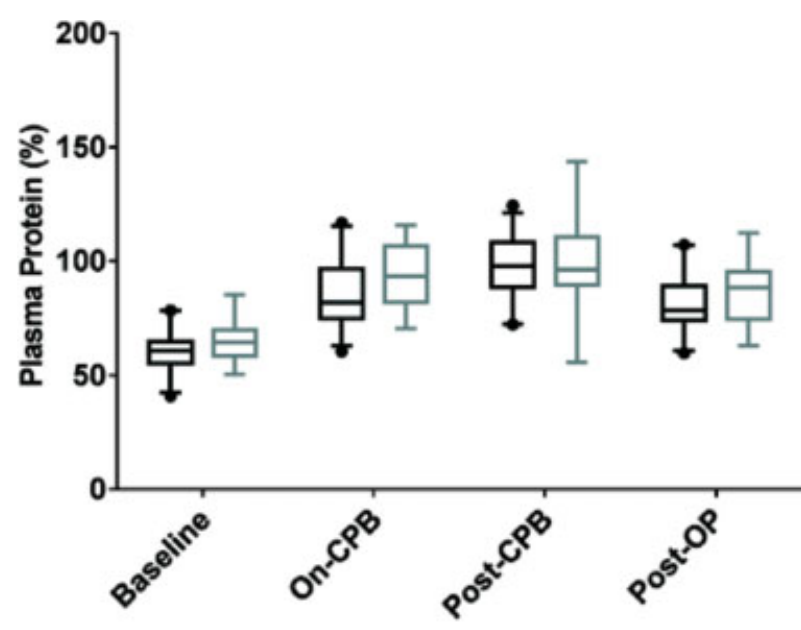

Fig. 1 Total plasma protein of the neonate increases upon transfusion of blood products at the beginning of surgery. Box and whisker plots for total plasma protein at the four surgical time points as per cent adult plasma are presented. The central line represents median, the boxes range from 25 th to 75 th percentiles, the whiskers extend from the 5 th to 95 th percentile and the dots depict outliers. There were no differences between the non-bleeder (black bars) and bleeder groups (grey bars) at any time point.

difference in plasma total protein between the bleeder and non-bleeder groups at any time point. Since the change in the concentration of each individual protein is associated with the change in total plasma protein concentration, the individual protein concentrations were modelled adjusting for the amount of total plasma protein in statistical analyses. However, the results of these analyses indicated that total plasma protein was not a confounding variable for changes observed in the concentration of individual plasma proteins.

\section{Change in Surgical Fibrinogen and D-Dimer}

Fibrinogen consumption and elevation of D-dimer suggest activation of coagulation and fibrinolytic cascades and have been related to the propensity for bleeding during paediatric
CPB. ${ }^{26}$ Average plasma fibrinogen was above $200 \mathrm{mg} / \mathrm{dL}$ at baseline and remained near or above this level throughout surgery in both the non-bleeder and bleeder groups as per the surgical transfusion protocol (-Fig. 2A). There were no differences in fibrinogen between the two groups. Plasma Ddimer was elevated at baseline, consistent with previous reports of neonates with congenital heart defects. ${ }^{27}$ It decreased from baseline at the post-CPB and post-op time points $(p<0.0001)$, perhaps reflecting dilution following transfusion or inhibition of fibrinolysis by tranexamic acid (-Fig. 2B). D-dimer was not measured at on-CPB due to interference from the high amounts of heparin present in the samples. There were no differences in D-dimer between the non-bleeder and bleeder groups at the three time points measured.

\section{Change in Surgical Plasma Anti-Thrombin Concentration and Conformation}

Anti-thrombin inhibits thrombin and FXa, as well as other proteases of the coagulation cascade in the presence of heparin. ${ }^{28}$ It was approximately 45 to $50 \%$ of the adult plasma concentration at baseline and fluctuated slightly higher during surgery (-Fig. 3A). The overall pattern of change mirrored that of total protein, but the anti-thrombin increase lagged total protein, suggesting consumption, particularly in on-CPB samples. There were no differences between the non-bleeder and the bleeder groups at any time point. Anti-thrombin TUG gel conformational analysis revealed primarily a fully active, virgin form at baseline (-Fig. 4). At later time points, a rapidly migrating band that did not unfold, representing proteolytically inactivated anti-thrombin, as well as multiple higher molecular weight bands that did not unfold, representing polymerized and protease complexed anti-thrombin emerged (-Fig. 4). These changes, most prevalent in on-CPB samples, were also present post-CPB and post-op. There were no identifiable patterns in anti-thrombin TUG gels consistently associated with non-bleeders or bleeders.
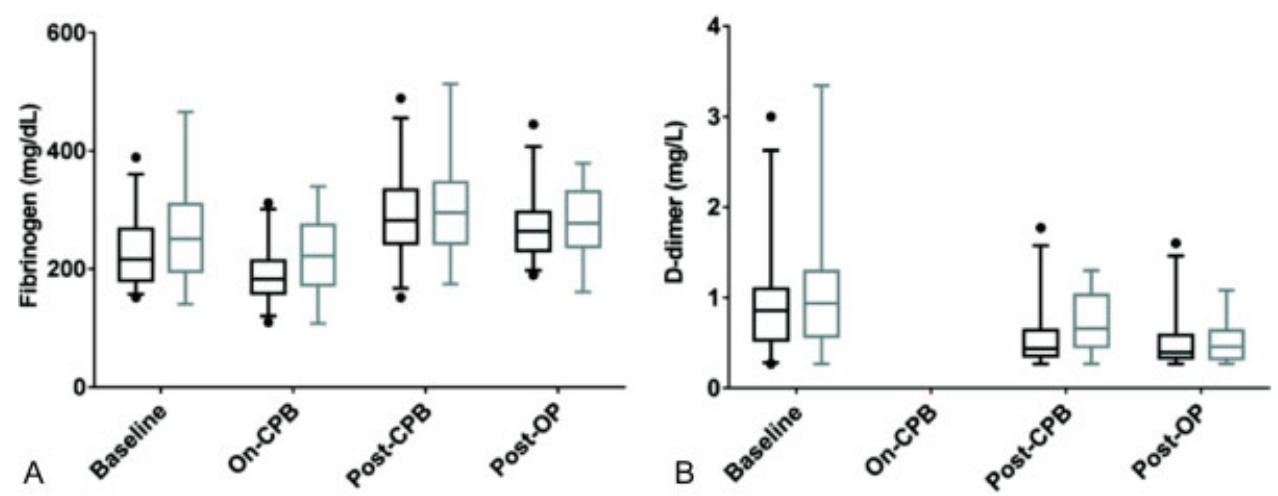

Fig. 2 Change in fibrinogen and D-dimer across surgery. Box and whisker plots as described in - Fig. 1 for fibrinogen and D-dimer concentration at the four surgical time points in the non-bleeder (black bars) and bleeder (grey bars) groups are presented. (A) Fibrinogen decreased from baseline to on-cardiopulmonary bypass (CPB), $p=0.0005$ for non-bleeder; $p=0.0041$ for bleeder. At post-CPB and post-op, fibrinogen increased compared with on-CPB, $p<0.0001$ for non-bleeder at both time points; $p<0.0001$ and $p=0.0009$ for bleeder at post-CPB and postop, respectively. (B) D-dimer decreased at post-CPB and post-op compared with baseline, $p<0.0001$ for both groups at both time points. Ddimer was not measured at on-CPB due to heparin interference with the assay. There were no differences in fibrinogen or D-dimer between the non-bleeder and bleeder groups at any time point, $p=0.1735$ and $p=0.3809$, respectively. 

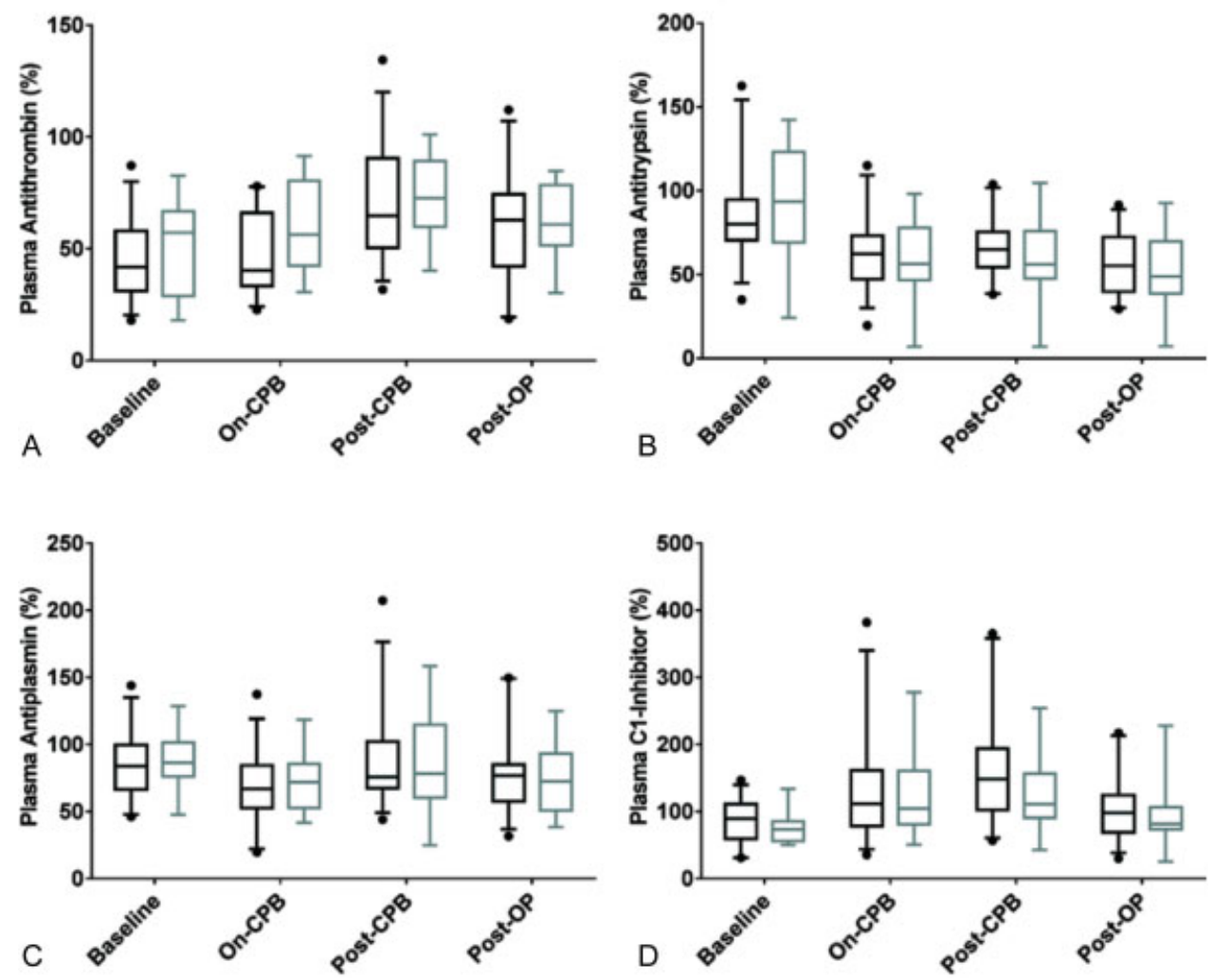

Fig. 3 Change in anti-thrombin, antitrypsin, anti-plasmin and C1-inhibitor across surgery. Box and whisker plots as described in $\mathbf{- F i g . ~} \mathbf{1}$ for plasma serpin concentrations at the four surgical time points as per cent adult plasma in the non-bleeder (black bars) and bleeder groups (grey bars) are presented. (A) Anti-thrombin was increased at post-cardiopulmonary bypass (CPB) compared with baseline and on-CPB, $p<0.0001$ for non-bleeders; $p=0.0009$ and $p=0.0386$ for bleeders. (B) Antitrypsin was decreased at all three surgical time points compared with baseline, $p<0.0001$ for the non-bleeder and bleeder groups. (C) Anti-plasmin decreased between baseline and on-CPB, $p=0.0006$ for non-bleeders; $p=0.0285$ for bleeders. (D) C1-inhibitor increased at on-CPB and post-CPB compared with baseline, $p<0.0001$ for the non-bleeder group at onCPB and post-CPB; $p<0.0001$ for the bleeder group at on-CPB and $p=0.0002$ at post-CPB, but was not different from baseline at post-op, $p=0.0864$ for non-bleeders and $p=0.2236$ for bleeders. There were no differences between the non-bleeder and bleeder groups at any time point for any serpin, $p>0.1203$ for all.

\section{Change in Surgical Plasma Antitrypsin Concentration and Conformation}

Antitrypsin plasma concentration is over $1.0 \mathrm{~g} / \mathrm{L}$, higher than other plasma protease inhibitor. As a rapid neutrophil elastase inhibitor, it protects tissues from enzymatic degradation during inflammation. ${ }^{29}$ The neonatal plasma concentration was approximately 85 to $95 \%$ of adults at baseline and decreased by 20 to $40 \%$ over time during surgery $(p<0.0001 ;$-Fig. 3B), suggesting consumption. No differences in antitrypsin levels between the non-bleeder and bleeder groups were found. Antitrypsin TUG gel conformational analysis revealed only the fully active, virgin form across surgery (-Fig. 4). Interestingly, in addition to the classical signature ( $\mathbf{- F i g . 5 A )},{ }^{19}$ three unique signatures were seen at baseline; a slightly less stable form that unfolded at low urea concentration (-Fig. 5B), a form that migrated slightly faster following unfolding at high urea concentration ( $\mathbf{- F i g}$. 5C) and a double heterozygous form combining these two less common forms ( - Fig. 5D). An association between the antitrypsin TUG gel baseline forms and non-bleeder versus bleeder group status was not identified.

\section{Change in Surgical Plasma Anti-Plasmin Concentration and Conformation}

Anti-plasmin is the primary plasma inhibitor of plasmin, the enzyme that degrades fibrin. ${ }^{30}$ It measured approximately 85 to $90 \%$ of the adult plasma concentration at baseline, with a fluctuating pattern across surgery ( - Fig. 3C). No differences between the non-bleeder and bleeder groups were found. Anti-plasmin TUG gel conformational analysis revealed only the fully active, virgin form across surgery (-Fig. 4). A minor band that migrated slightly faster than the anti-plasmin primary form was also present in all samples across surgery. This minor band appears to reflect the heterogeneity of plasma anti-plasmin at both its $\mathrm{N}$ - and Ctermini. $^{31-33}$

\section{Change in Surgical Plasma Anti-Chymotrypsin Conformation}

Anti-chymotrypsin is an inhibitor of neutrophil cathepsin G and limits tissue injury at sites of inflammation. ${ }^{29}$ Measurement of anti-chymotrypsin plasma concentration by ELISA was not performed, because we were unable to identify an antibody pair that worked consistently. Anti-chymotrypsin 

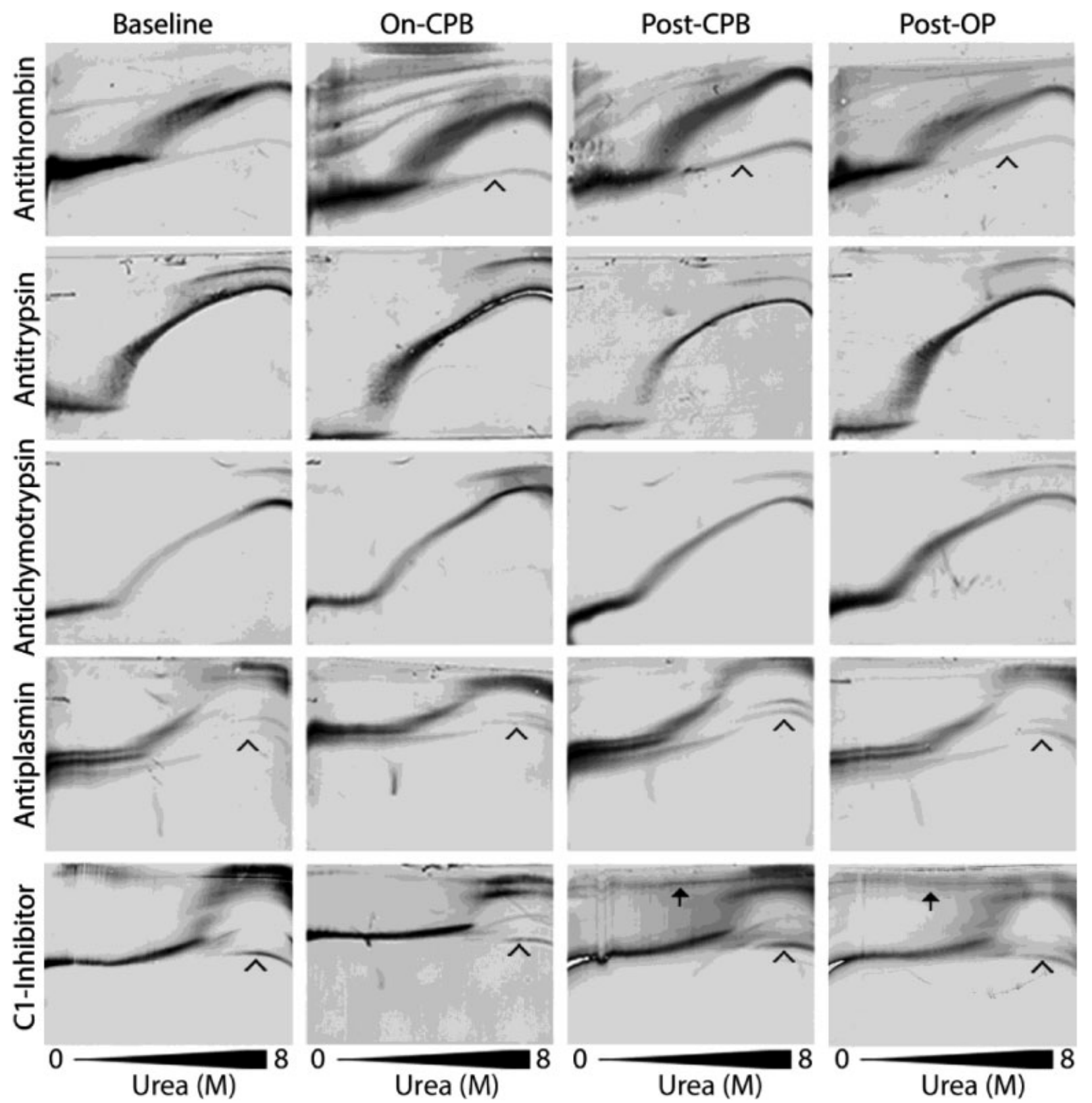

Fig. 4 Transverse urea gradient (TUG) gel electrophoresis with Western blot to assess conformational stability of serpins across neonatal cardiopulmonary bypass (CPB). Each panel represents a single gel with migration from top to bottom and 0-8 $\mathrm{M}$ urea gradient from left to right. There were minor differences for different serpins at the various time points observed in some patients, but overall results were consistent. The most representative gels from individual neonates are presented. All serpins exhibited the virgin conformation at baseline. There are minor forms of anti-plasmin and C1-inhibitor that unfold differently (arrowheads) than the primary form. Other than anti-thrombin and C1-inhibitor, the serpins remained in virgin conformation across surgery. New conformations of anti-thrombin appeared in samples collected during surgery. These include reactive site cleaved anti-thrombin present as a rapidly migrating band that did not unfold in $8 \mathrm{M}$ urea (arrowhead), as well as multiple slower migrating bands, that represent anti-thrombin-protease complexes, or polymerized protein. A new form of slowly migrating C1inhibitor, likely representing a protease-complexed form, was observed at post-CPB and post-op (arrows).

TUG gel conformational analysis revealed only the fully active, virgin form across surgery (-Fig. 4) with no differences between the non-bleeder and bleeder groups.

\section{Change in Surgical Plasma C1-Inhibitor Concentration and Conformation}

C1-inhibitor inhibits several proteases of the complement cascade. $^{34}$ It was approximately 85 to $90 \%$ of the adult plasma concentration at baseline, increased to 45 to $75 \%$ in on-CPB and post-CPB samples, before returning towards baseline at post-op ( - Fig. 3D). There were no differences between the non-bleeder and bleeder groups across surgery. C1-inhibitor TUG gel conformational analysis revealed primarily the fully active, virgin form at baseline ( - Fig. 4 ). There were several minor bands on the C1-inhibitor TUG gels that were most apparent at post-CPB and post-op (- Fig. 4). One 

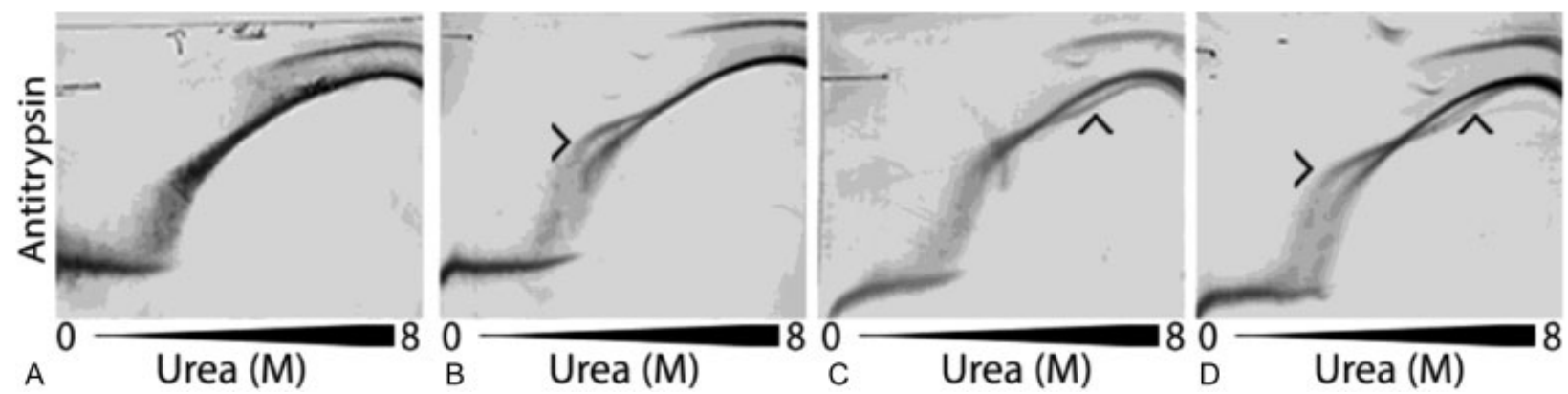

Fig. 5 Transverse urea gradient (TUG) gel electrophoresis and Western blot reveals unique unfolding signatures of virgin antitrypsin present in different neonates at baseline. (A) The classical signature; (B) A slightly less stable form that unfolded at low urea concentration (arrowhead); (C) A form that migrated slightly faster following unfolding at high urea concentration (arrowhead); and (D) a neonate heterozygous for the two variant forms (arrowheads).

band unfolded in a similar pattern to virgin $\mathrm{C} 1$-inhibitor and may represent a form that has undergone $\mathrm{N}$ - or $\mathrm{C}$-terminal proteolysis or a form with altered glycosylation. ${ }^{35}$ There also were high molecular weight bands that did not unfold, a pattern consistent with circulating $\mathrm{C} 1$-inhibitor-protease complex and low level consumption. An association between the presence or apparent amount of C1-inhibitor-protease complex and bleeding status was not identified.

\section{Change in Surgical Plasma TFPI Concentration and Degradation}

TFPI, a Kunitz-type protease inhibitor, dampens early stages of a pro-coagulant response by inhibiting tissue factor, FVIIa in a FXa-dependent manner, ${ }^{36}$ and early forms of prothrombinase, the catalytic complex containing FXa and FVa. ${ }^{37}$ Plasma TFPI is present in the full-length form, termed TFPI $\alpha$, and C-terminally truncated forms. ${ }^{38}$ Plasma total TFPI (TFPI $\alpha$ plus truncated TFPI) and TFPI $\alpha$ concentrations were measured across surgery. At baseline, total TFPI was approximately 20 to $25 \%$ the adult concentration with TFPI $\alpha$ representing approximately $10 \%$ of the total plasma TFPI ( - Fig. 6A). Total TFPI and TFPI $\alpha$ promptly increase two- to fourfold upon heparin infusion in adults. ${ }^{39,40}$ Heparin-relea- sable TFPI was similarly observed in neonates, as total TFPI increased over twofold between baseline and on-CРB. At post-CPB and post-op, it declined slightly but remained elevated at twofold over baseline. TFPI $\alpha$ increased fivefold between baseline and on-CPB ( $\mathbf{- F i g . ~ 6 B ) . ~ I t ~ t h e n ~ d e c l i n e d ~ t o ~}$ about fourfold over baseline at post-CPB and twofold at postop. Despite these large changes in plasma TFPI across surgery, there were no differences in the plasma concentration of either total TFPI or TFPI $\alpha$ between the non-bleeder and bleeder groups. TFPI Western blots ( - Fig. $\mathbf{6 C}$ ) mirror the ELISA data with greatly increased band intensity in samples collected after baseline. Of note, the presence of a small amount of degraded TFPI apparent at post-CPB and post-op may partially explain the continued elevation of plasma TFPI following reversal of heparin with protamine in the post- $\mathrm{CPB}$ and post-op samples.

\section{Discussion}

Physiological responses to the haemostatic stress of CPB were evaluated by examining changes in concentration and structural conformation of plasma protease inhibitors regulating the coagulation, inflammatory, fibrinolytic and
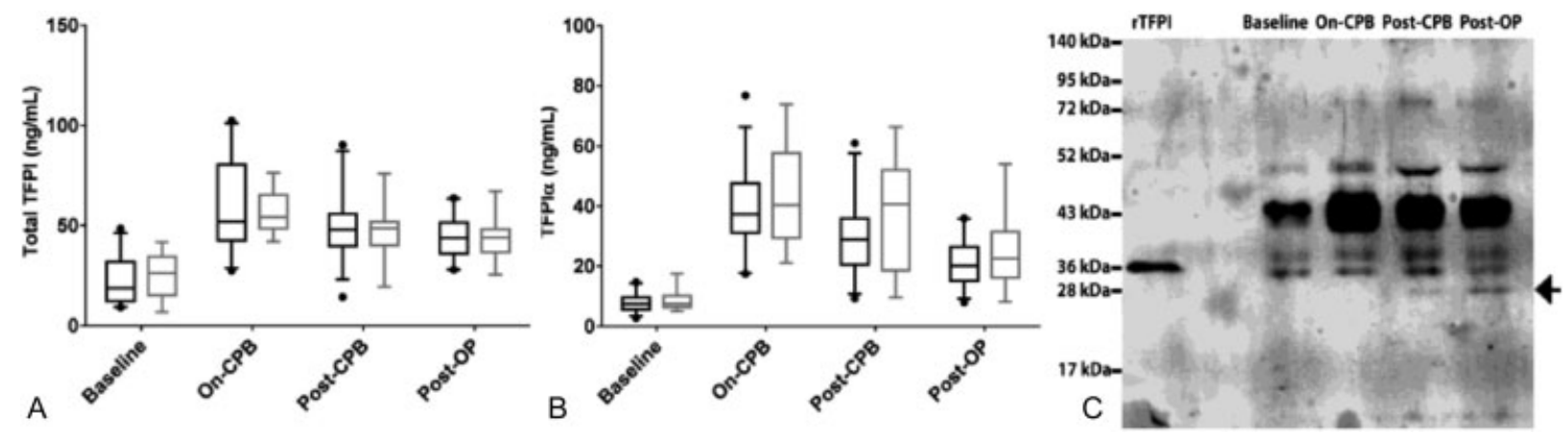

Fig. 6 Change in tissue factor pathway inhibitor (TFPI) concentration and partial degradation across surgery. In panels A and B, box and whisker plots as described in - Fig. 1 with black bars representing the non-bleeding group and grey bars the bleeding group are presented. (A) Total TFPI increased over twofold in on-cardiopulmonary bypass (CPB) samples compared with baseline and remained twofold elevated across surgery, $p<0.0001$. There were no differences in total TFPI or TFPl $\alpha$ between the non-bleeder and bleeder groups at any time point, $p=0.9353$. (B) TFPl $\alpha$ increased fivefold in on-CPB samples compared with baseline and remained over fourfold elevated across surgery, $p<0.0001$. There were no differences in total TFPI or TFPI $\alpha$ between the non-bleeder and bleeder groups at any time point, $p=0.1486$. (C) TFPI Western blot demonstrates a marked increase in the amount of plasma TFPI after baseline. Degraded forms of TFPI were present in post-CPB and post-op samples (arrow). 
complement cascades in neonates undergoing complex cardiac surgical procedures. TUG gel analysis identifies these different serpin conformations in plasma in a simple, low cost manner that requires only micro-litres of plasma. ${ }^{19,20}$ These analyses were used to obtain 'snapshots' of serpin conformational changes occurring across the cardiac surgical procedures. Although a decrease in fibrinogen concentration was not observed, large effects on the structural conformation of anti-thrombin were observed, indicating activation of the coagulation cascade. The heparin infusion occurring between baseline and on-CPB was associated with the accumulation of non-virgin forms of anti-thrombin in plasma. These remained following protamine infusion at post- $\mathrm{CPB}$ or post-op, suggesting continued activation of coagulation proteases at the end of surgery. However, no associations were identified between change in plasma concentration or structure of anti-thrombin and post-operative bleeding. Despite activation of the blood coagulation cascade, only minimal conformational change of other protease inhibitors was observed. Thus, based on serpin conformational change, there was no evidence for substantial CPB-mediated activation or cross-activation by coagulation proteases of other plasma proteolytic cascades in the neonates. Further, ample amounts of the virgin form of each serpin examined were present across surgery indicating that widespread or uncontrolled proteolysis did not occur, even in the patients experiencing bleeding.

The presence of the inactive forms of anti-thrombin in on$\mathrm{CPB}$, post-CPB and post-op samples indicates that ELISA measurement of antigen likely over-estimates the active anti-thrombin in plasma. However, examination of the TUG gels revealed that typically $75 \%$ or more of the antithrombin remained in the virgin conformation and severe depletion of active anti-thrombin was never observed. These data suggest that the neonates had ample anti-thrombin, either endogenous or transfused, and would not have further benefitted from infusion of anti-thrombin concentrate.

TFPI $\alpha$ is a heparin-releasable plasma protein in adults. ${ }^{39,40}$ The neonates had a similar response to heparin, as plasma TFPI $\alpha$ increased an average of fivefold between baseline and on-CPB. The neonates differed from adult CPB patients in that TFPI $\alpha$ remained elevated over twofold following protamine reversal in the post-CPB and post-op samples, while it returned to baseline in adults. ${ }^{41}$ Similar to adults, C-terminal degradation of a small portion of circulating TFPI $\alpha$ occurred during surgery. ${ }^{41}$ One possible cause for the elevated TFPI remaining in the circulation would be incomplete protamine reversal of heparin. However, it may also result from a unique interaction between neonatal TFPI and vasculature that is not present in adults. Further studies are needed to understand this interaction. TFPI is a Kunitz-type protease inhibitor with inhibitory domains defined by disulphide bonds; therefore, unfolding transitions were not examined using TUG gel conformation analysis. While the elevated plasma TFPI $\alpha$ at the end of surgery is expected to increase plasma anticoagulant activity, it remains of uncertain physiological significance because it was not associated with post-operative bleeding in the neonates.
Although previous studies have found activation of complement, and inflammatory cascades in paediatric $\mathrm{CPB},{ }^{11}$ there was remarkably little change in the concentration or structural conformation of the serpins regulating inflammatory, fibrinolytic and complement proteolytic cascades. Plasma concentrations of antitrypsin, anti-plasmin and C1-inhibitor each remained at $60 \%$ or more of baseline with only small amounts of non-virgin conformations observed in TUG gels, providing little evidence for extensive unregulated cross-activation of non-coagulation proteolytic cascades by coagulation proteases or cascade activation by exposure to artificial surfaces in the bypass pump. Of the changes observed, perhaps the most relevant was the plasma antitrypsin concentration. Antitrypsin is an acute phase reactant and, therefore, may have been expected to increase across surgery. Instead, it decreased 20 to $40 \%$ from baseline, suggesting the possibility of consumption. It is possible that there was rapid clearance of antitrypsin-protease complexes from the circulation, as only the virgin form was observed on TUG gel conformational analysis, but antitrypsin-protease complexes are cleared from the circulation by the same receptor as anti-thrombin-protease complexes, ${ }^{21}$ which were observed in the TUG gels. Thus, the mechanism for the decrease in the plasma antitrypsin concentration remains unclear.

In contrast to antitrypsin, C1-inhibitor, which also is an acute phase reactant, was increased in on- $\mathrm{CPB}$ and post-CPB samples. There was a small amount of protease-complexed C1-inhibitor in samples collected after baseline, but over $90 \%$ remained in the virgin conformation. The increase in C1inhibitor observed here contrasts with a previous study where a significant drop was observed in $<12$-month-old patients undergoing СРB. ${ }^{42}$ However, several of these babies experienced capillary leak syndrome, which is associated with the decreased C1-inhibitor and did not develop in neonates studied here.

In addition to identifying serpin conformational changes occurring across surgery, the TUG gels performed using baseline samples uncovered natural variants in antitrypsin, anti-plasmin and C1-inhibitor. Antitrypsin had two altered unfolding patterns, one at lower and one at higher urea concentrations. Neonates having neither, either or both patterns at baseline were identified, suggesting normal polymorphic variation in the protein, although altered glycosylation may also cause these variants. Further studies are needed to biochemically define these variants and how they alter the structural stability of antitrypsin. Anti-plasmin and C1-inhibitor had minor bands that unfolded in a non-classical pattern in TUG gels. These were identically present in all samples and may represent partial $\mathrm{N}$ - or C-terminal variants or degradation or altered glycosylation. ${ }^{31-33,35}$

A limitation to this study is that excessive bleeding in some patients with post-operative bleeding began in the operating room, as evidenced by the increased number of blood products they received during surgery. To account for this, the analyses comparing protein concentrations in nonbleeders to bleeders were adjusted for the amount of total protein in each plasma sample to account for the effects of 
transfusion, but this did not alter the changes observed for the individual plasma protein concentrations. Thus, while transfusion produced a major change in plasma protein concentration at the beginning of surgery, the transfusions administered in response to patient bleeding that occurred primarily in the post-CPB period had minimal impact on plasma protein concentration. However, the intra-operative transfusions may have masked significant declines in the plasma protease inhibitors among the bleeding patients. Similarly, the transfusions may have impacted the conformations of the serpins observed on the TUG gels, particularly the virgin forms, which are in FFP. ${ }^{43}$ However, the consistent presence of non-virgin forms of anti-thrombin across the surgical procedure in all neonates suggests that if other serpins existed in non-virgin conformations, they also would have been observed on the TUG gels.

None of the protease inhibitor plasma concentrations or conformational changes were associated with post-operative bleeding in the neonates, reflecting the multiple possible causes of bleeding in this population with a complex disease undergoing a complex surgical procedure. Direct comparisons of the findings presented here to those of previous studies of neonatal or paediatric CPB are difficult due to variations in study protocols and patient management. These include differences in the age of the patients enrolled, exclusion criteria, priming solutions and differences in transfusion of blood products, colloid solutions and recombinant anti-thrombin. Therefore, our findings and conclusions about cross-activation of plasma proteolytic cascades by coagulation proteases and the effects of changes in plasma protease inhibitor concentration and conformation on post-operative bleeding following neonatal CPB are applicable to patients treated with a transfusion protocol similar to that used here.

\section{What is known about this topic?}

- Exposure of blood to artificial materials during cardiopulmonary bypass can activate coagulation, complement and inflammatory pathways.

- Serpin conformation can be readily detected using transverse urea gradient gels and serve as a plasma biosensor for proteolytic cascade activation.

- Tissue factor pathway inhibitor is a heparin-releasable protein.

\section{What does this paper add?}

- Blood samples from 44 neonates were obtained at four times points during cardiopulmonary bypass surgery.

- Coagulation was activated as evidenced by changes in the conformation of anti-thrombin, but extensive cross-activation of other cascades was not observed.

- Plasma tissue factor pathway inhibitor increased upon heparin infusion and remained elevated following protamine administration. However, it was not associated with bleeding in this population.

\section{Authors' Contributions}

S.A. Maroney: designed research, performed experiments, interpreted data and wrote the manuscript. J.A. Peterson: designed research, interpreted data and wrote the manuscript. W. Zwifelhofer: performed experiments. N. D. Martinez: performed experiments. K. Yan: interpreted data and performed statistical analysis. R.S. Bercovitz: designed research and interpreted data. R.K. Woods: performed surgery, designed research and interpreted data. A.E Mast: designed research, interpreted data and wrote the manuscript.

\section{Funding}

This work was supported by National Heart, Lung, and Blood Institute grant HL068835 (to A.E. Mast) and the Herma Heart Center at Children's Hospital of Wisconsin.

\section{Conflict of Interest}

A.E. Mast receives grant support from Novo Nordisk. The other authors have no conflicts to declare.

\section{References}

1 Arnold PD. Coagulation and the surgical neonate. Paediatr Anaesth 2014;24(01):89-97

2 Whiting D, Yuki K, DiNardo JA. Cardiopulmonary bypass in the pediatric population. Best Pract Res Clin Anaesthesiol 2015;29 (02):241-256

3 Ferrer-Marin F, Stanworth S, Josephson C, Sola-Visner M. Distinct differences in platelet production and function between neonates and adults: implications for platelet transfusion practice. Transfusion 2013;53(11):2814-2821

4 Hézard N, Potron G, Schlegel N, Amory C, Leroux B, Nguyen P. Unexpected persistence of platelet hyporeactivity beyond the neonatal period: a flow cytometric study in neonates, infants and older children. Thromb Haemost 2003;90(01):116-123

5 Rajasekhar D, Kestin AS, Bednarek FJ, Ellis PA, Barnard MR, Michelson AD. Neonatal platelets are less reactive than adult platelets to physiological agonists in whole blood. Thromb Haemost 1994;72(06):957-963

6 Cade JF, Hirsh J, Martin M. Placental barrier to coagulation factors: its relevance to the coagulation defect at birth and to haemorrhage in the newborn. BMJ 1969;2(5652):281-283

7 Andrew M, Paes B, Milner R, et al. Development of the human coagulation system in the full-term infant. Blood 1987;70(01): 165-172

8 Reverdiau-Moalic P, Delahousse B, Body G, Bardos P, Leroy J, Gruel Y. Evolution of blood coagulation activators and inhibitors in the healthy human fetus. Blood 1996;88(03):900-906

9 Strauss T, Sidlik-Muskatel R, Kenet G. Developmental hemostasis: primary hemostasis and evaluation of platelet function in neonates. Semin Fetal Neonatal Med 2011;16(06): 301-304

10 Journois D, Pouard P, Greeley WJ, Mauriat P, Vouhé P, Safran D. Hemofiltration during cardiopulmonary bypass in pediatric cardiac surgery. Effects on hemostasis, cytokines, and complement components. Anesthesiology 1994;81(05):1181-1189

11 Sonntag J, Dähnert I, Stiller B, Hetzer R, Lange PE. Complement and contact activation during cardiovascular operations in infants. Ann Thorac Surg 1998;65(02):525-531

12 Sato H, Yamamoto K, Kakinuma A, Nakata Y, Sawamura S. Accelerated activation of the coagulation pathway during cardiopulmonary bypass in aortic replacement surgery: a prospective observational study. J Cardiothorac Surg 2015;10:84 
13 Sniecinski RM, Chandler WL. Activation of the hemostatic system during cardiopulmonary bypass. Anesth Analg 2011;113(06): 1319-1333

14 Morizumi S, Hiramatsu Y, Matsuzaki K, et al. Early heparin administration attenuates tissue factor-mediated thrombin generation during simulated cardiopulmonary bypass. J Card Surg 2014;29(01):35-40

15 Ignjatovic V, Than J, Summerhayes R, et al. Hemostatic response in paediatric patients undergoing cardiopulmonary bypass surgery. Pediatr Cardiol 2011;32(05):621-627

16 Foley JH, Conway EM. Cross talk pathways between coagulation and inflammation. Circ Res 2016;118(09):1392-1408

17 Ge X, Yamaguchi Y, Zhao L, et al. Prochemerin cleavage by factor XIa links coagulation and inflammation. Blood 2018;131(03): 353-364

18 Loebermann H, Tokuoka R, Deisenhofer J, Huber R. Human alpha 1-proteinase inhibitor. Crystal structure analysis of two crystal modifications, molecular model and preliminary analysis of the implications for function. J Mol Biol 1984;177(03):531-557

19 Mast AE, Enghild JJ, Pizzo SV, Salvesen G. Analysis of the plasma elimination kinetics and conformational stabilities of native, proteinase-complexed, and reactive site cleaved serpins: comparison of alpha 1-proteinase inhibitor, alpha 1-antichymotrypsin, antithrombin III, alpha 2-antiplasmin, angiotensinogen, and ovalbumin. Biochemistry 1991;30(06):1723-1730

20 Mast AE, Enghild JJ, Salvesen G. Conformation of the reactive site loop of alpha 1-proteinase inhibitor probed by limited proteolysis. Biochemistry 1992;31(10):2720-2728

21 Pizzo SV, Mast AE, Feldman SR, Salvesen G. In vivo catabolism of alpha 1-antichymotrypsin is mediated by the Serpin receptor which binds alpha 1-proteinase inhibitor, antithrombin III and heparin cofactor II. Biochim Biophys Acta 1988;967(02):158-162

22 Bercovitz RS, Shewmake AC, Newman DK, et al. Validation of a definition of excessive postoperative bleeding in infants undergoing cardiac surgery with cardiopulmonary bypass. J Thorac Cardiovasc Surg 2018;155(05):2112-2124

23 Dietzen DJ, Jack GG, Page KL, Tetzloff TA, Hall CL, Mast AE. Localization of tissue factor pathway inhibitor to lipid rafts is not required for inhibition of factor VIIa/tissue factor activity. Thromb Haemost 2003;89(01):65-73

24 Ellery PER, Hilden I, Sejling K, et al. Correlates of plasma and platelet tissue factor pathway inhibitor, factor $\mathrm{V}$, and protein $\mathrm{S}$. Res Pract Thromb Haemost 2018;2(01):93-104

25 Maroney SA, Haberichter SL, Friese P, et al. Active tissue factor pathway inhibitor is expressed on the surface of coated platelets. Blood 2007;109(05):1931-1937

26 Faraoni D, Willems A, Savan V, Demanet H, De Ville A, Van der Linden P. Plasma fibrinogen concentration is correlated with postoperative blood loss in children undergoing cardiac surgery. A retrospective review. Eur J Anaesthesiol 2014;31(06):317-326

27 Hakacova N, Laluhova-Striezencova Z, Zahorec M. Disturbances of coagulation in neonates with functionally univentricular physiol- ogy prior to the first stage of surgical reconstruction. Cardiol Young 2008;18(04):397-401

28 Damus PS, Hicks M, Rosenberg RD. Anticoagulant action of heparin. Nature 1973;246(5432):355-357

29 Travis J, Salvesen GS. Human plasma proteinase inhibitors. Annu Rev Biochem 1983;52:655-709

30 Collen D. Identification and some properties of a new fastreacting plasmin inhibitor in human plasma. Eur J Biochem 1976;69(01):209-216

31 Bangert K, Johnsen AH, Christensen U, Thorsen S. Different Nterminal forms of alpha 2-plasmin inhibitor in human plasma. Biochem J 1993;291(Pt 2):623-625

32 Koyama T, Koike Y, Toyota S, Miyagi F, Suzuki N, Aoki N. Different $\mathrm{NH} 2$-terminal form with 12 additional residues of alpha 2-plasmin inhibitor from human plasma and culture media of Hep G2 cells. Biochem Biophys Res Commun 1994;200(01):417-422

33 Kluft C, Los N. Demonstration of two forms of alpha 2-antiplasmin in plasma by modified crossed immunoelectrophoresis. Thromb Res 1981;21(1-2):65-71

34 Davis AE III, Whitehead AS, Harrison RA, et al. Human inhibitor of the first component of complement, $\mathrm{C} 1$ : characterization of cDNA clones and localization of the gene to chromosome 11. Proc Natl Acad Sci U S A 1986;83(10):3161-3165

35 Schoenberger OL, Sprows JL, Schechter NM, Cooperman BS, Rubin $\mathrm{H}$. Limited proteolysis of $\mathrm{C} 1$-inhibitor by chymotrypsin-like proteinases. FEBS Lett 1989;259(01):165-167

36 Rao LV, Rapaport SI. Studies of a mechanism inhibiting the initiation of the extrinsic pathway of coagulation. Blood 1987; 69(02):645-651

37 Wood JP, Bunce MW, Maroney SA, Tracy PB, Camire RM, Mast AE. Tissue factor pathway inhibitor-alpha inhibits prothrombinase during the initiation of blood coagulation. Proc Natl Acad Sci U S A 2013;110(44):17838-17843

38 Broze GJ Jr, Lange GW, Duffin KL, MacPhail L. Heterogeneity of plasma tissue factor pathway inhibitor. Blood Coagul Fibrinolysis 1994;5(04):551-559

39 Novotny WF, Palmier M, Wun TC, Broze GJ Jr, Miletich JP. Purification and properties of heparin-releasable lipoprotein-associated coagulation inhibitor. Blood 1991;78(02):394-400

40 Sandset PM, Abildgaard U, Larsen ML. Heparin induces release of extrinsic coagulation pathway inhibitor (EPI). Thromb Res 1988; 50(06):803-813

41 Donahue BS, Gailani D, Mast AE. Disposition of tissue factor pathway inhibitor during cardiopulmonary bypass. J Thromb Haemost 2006;4(05):1011-1016

42 Stiller B, Sonntag J, Dähnert I, et al. Capillary leak syndrome in children who undergo cardiopulmonary bypass: clinical outcome in comparison with complement activation and $\mathrm{C} 1$ inhibitor. Intensive Care Med 2001;27(01):193-200

43 Mast AE, Stadanlick JE, Lockett JM, Dietzen DJ. Solvent/detergenttreated plasma has decreased antitrypsin activity and absent antiplasmin activity. Blood 1999;94(11):3922-3927 